\title{
Employing of CRISPR/Cas9 technology to knock out genes associated with flowering in aspen
}

\author{
Karzhaev D.S. \\ Saint-Petersburg Forestry Research Institute, St. Petersburg, Russia \\ email: karzhaevd@gmail.com
}

\begin{abstract}
Populus tremula L. (aspen) is a widespread tree species that is extensively used in urban landscaping and forestry plantations due to its high growth rate. However, some undesirable traits, such as vulnerability to fungal pathogens, or the formation of "fluff", which are produced by the female trees of this species, causing allergic reactions, pose problems for the propagating of aspen. In order to improve some of the aspen disadvantages the gene-editing methods could be employed. To date, due to the risk of gene transfer from genetically edited plants to native ecosystems, these biotechnological approaches have not been widely used in forestry, and their implementation faces many limitations. To overcome these limitations and apply the gene editing techniques to woody plants, a project focusing on CRISPR/Cas9 knockout of flowering-related genes in aspen was initiated, aiming to create sterile plants. In early studies, many genes associated with flowering were identified in aspen, and further experiments were carried out to reduce the expression of these genes using RNA interference. In order to fix the sterility trait in the aspen genotype, we selected the 3 most promising genes to knockout and created sgRNA for the targets. Callus cultures of aspen will be treated with bioballistic method, the obtained regenerates will serve as a base for further gene editing experiments, focusing on manipulation of genes responsible for productivity, resistance and wood quality.
\end{abstract}

